\title{
Poorly Controlled Congenital Hypothyroidism due to an Underlying Allgrove Syndrome
}

\author{
V. van Tellingen ${ }^{a}$ M.J.J. Finken ${ }^{b} \quad$ J. Israëls ${ }^{c}$ Y.M.C. Hendriks ${ }^{d}$ G.A. Kamp ${ }^{e}$ \\ H.M. van Santen ${ }^{f}$ \\ ${ }^{a}$ Department of Pediatric Endocrinology, Emma Children's Hospital, Academic Medical Center, and \\ Departments of ${ }^{b}$ Pediatric Endocrinology, ${ }^{c}$ Pediatrics and ${ }^{d}$ Clinical Genetics, VU University Medical Center, \\ Amsterdam, e Department of Pediatrics, Tergooi Hospitals, Blaricum, and ${ }^{\mathrm{f} D e p a r t m e n t}$ of Pediatric Endocrinology, \\ Wilhelmina Children's Hospital, University Medical Center Utrecht, Utrecht, The Netherlands
}

\section{Established Facts}

- In patients with primary hypothyroidism, by far the most common cause of a persistently elevated thyrotropin level is poor adherence to thyroid hormone substitution therapy.

\section{Novel Insights}

- In patients with primary hypothyroidism and a persistently elevated thyrotropin level despite adequate thyroid hormone substitution therapy, factors that could cause this, although rare, should be systematically excluded (e.g., malabsorption and adrenal insufficiency).

- The causes of primary adrenal insufficiency are numerous. To speed up the diagnostic workup, specific signs and symptoms in proband and family members should be screened for.

\section{Key Words}

Congenital hypothyroidism · Thyroid hormone metabolism . Thyroid-stimulating hormone $\cdot$ Receptors $\cdot$ L-Thyroxine $\cdot$ Adrenal insufficiency - Thyrotropin receptor mutation . Levothyroxine treatment - Allgrove syndrome

\section{Abstract \\ Background: Congenital hypothyroidism of thyroidal origin (CHT) is a common disorder in pediatric endocrinology prac- tices, which can be difficult to manage. Elevated thyrotropin (TSH) concentrations are in the great majority of cases ex-}

\section{KARGER}

E-Mail karger@karger.com www.karger.com/hrp

\section{The Author(s) \\ Published by S. Karger AG, Basel \\ Karger \\ Open access}

This article is licensed under the Creative Commons Attribution NonCommercial-NoDerivatives 4.0 International License (CC BY NC-ND) (http://www.karger.com/Services/OpenAccessLicense). Usage and distribution for commercial purposes as well as any distribution of modified material requires written permission. plained by poor compliance to levothyroxine therapy. Methods: Case description. Results: We present a boy with CHT, with 2 heterozygous mutations in the TSH receptor gene, who showed persistently elevated TSH concentrations and psychomotor retardation, initially misinterpreted as malcompliance. At the age of 4 years, he was diagnosed with adrenal insufficiency, wherefore a broad diagnostic search was initiated. After the start of glucocorticoid replacement therapy, his TSH normalized and the levothyroxine could be lowered. At the age of 6 years, his TSH increased again, this time caused by malabsorption of levothyroxine due to esophageal achalasia. In retrospect, alacrima was also pres-
$\mathrm{V}$. van Tellingen, $\mathrm{MD}$

Department of Pediatric Endocrinology, Emma Children's Hospital Academic Medical Center, room No. H7-228, Meibergdreef 9

NL-1105 AZ Amsterdam (The Netherlands)

E-Mail v.vantellingen@amc.nl 
ent and the diagnosis of Allgrove syndrome was genetically confirmed. The $\mathrm{CHT}$ was considered a separate disease entity. Conclusions: In case of persistently elevated TSH levels in children with $\mathrm{CHT}$, causes other than noncompliance must be considered. Second, in establishing the cause of adrenal insufficiency, specific symptoms, such as alacrima, are easily overlooked. Third, Allgrove syndrome is a rare disorder, in which diagnostic delay can lead to potentially life-threatening complications.

(C) 2016 The Author(s)

Published by S. Karger AG, Basel

\section{Introduction}

Congenital hypothyroidism of thyroidal origin (CHT) is a common disorder in pediatric endocrinology practices and is characterized by an elevated concentration of thyrotropin (TSH) in combination with a low, or lownormal, concentration of free thyroxine T4 (FT4). Factors as linear growth, changes in body composition and difficulties in adherence to levothyroxine therapy, make disease control in children challenging [1]. After the start of levothyroxine substitution, in most cases, a quick normalization of TSH is observed. If TSH does not respond to levothyroxine as expected, this should be explored.

In this case report, we describe the pitfalls in the diagnostic process in a boy who presented with poorly controlled CHT. He exhibited a delay in psychomotor devel- opment, which was initially attributed to poor adherence to the levothyroxine supplementation. His TSH level was difficult to normalize. Following an acute deterioration at the age of 4 years, alternative explanations for the persistently elevated TSH were found: adrenal insufficiency and malabsorption due to Allgrove syndrome (see fig. 1 for an overview of the clinical course of our patient).

\section{Case Description}

A 2.5-year-old boy was seen at the outpatient ward of a regional hospital after moving to the Netherlands. The past medical history of the boy revealed CHT, which was diagnosed in Denmark through the neonatal screening program. In contrast to the Netherlands, Denmark follows a TSH-based screening. A TSH level $>25$ $\mathrm{mIU} / \mathrm{l}$ is considered a positive screening result. Levothyroxine therapy was started shortly after birth. The boy's parents were consanguineous to the second degree (first cousins). The family history revealed an older brother who had died unexpectedly in Morocco at the age of 2 years during an episode of gastroenteritis. No autopsy had been performed and the cause of death was never established.

On initial evaluation, after arrival in the Netherlands, he had a TSH concentration of $42 \mathrm{mIU} / \mathrm{l}$ (reference range: $0.5-4.5 \mathrm{mIU} / \mathrm{l}$ ) and an FT4 concentration of $20 \mathrm{pmol} / \mathrm{l}$ (reference range: 10-23 $\mathrm{pmol} / \mathrm{l}$ ), which raised questions about his compliance. Between 2.5 and 4 years of age, his TSH fluctuated between $<0.01$ and $23 \mathrm{mIU} / \mathrm{l}$, in spite of attempts to optimize the substitution dose of levothyroxine. To establish the etiology of the $\mathrm{CHT}$, further tests were done. He was thyroid peroxidase (TPO) antibody negative, and the thyroglobulin concentration was normal. An ultrasound of the
Fig. 1. Clinical course of our patient with a line representing changes in TSH over time (reference interval between dotted lines) and doses of levothyroxine and hydrocortisone showed as bars of variable height. Important events in the medical history are indicated by arrows.

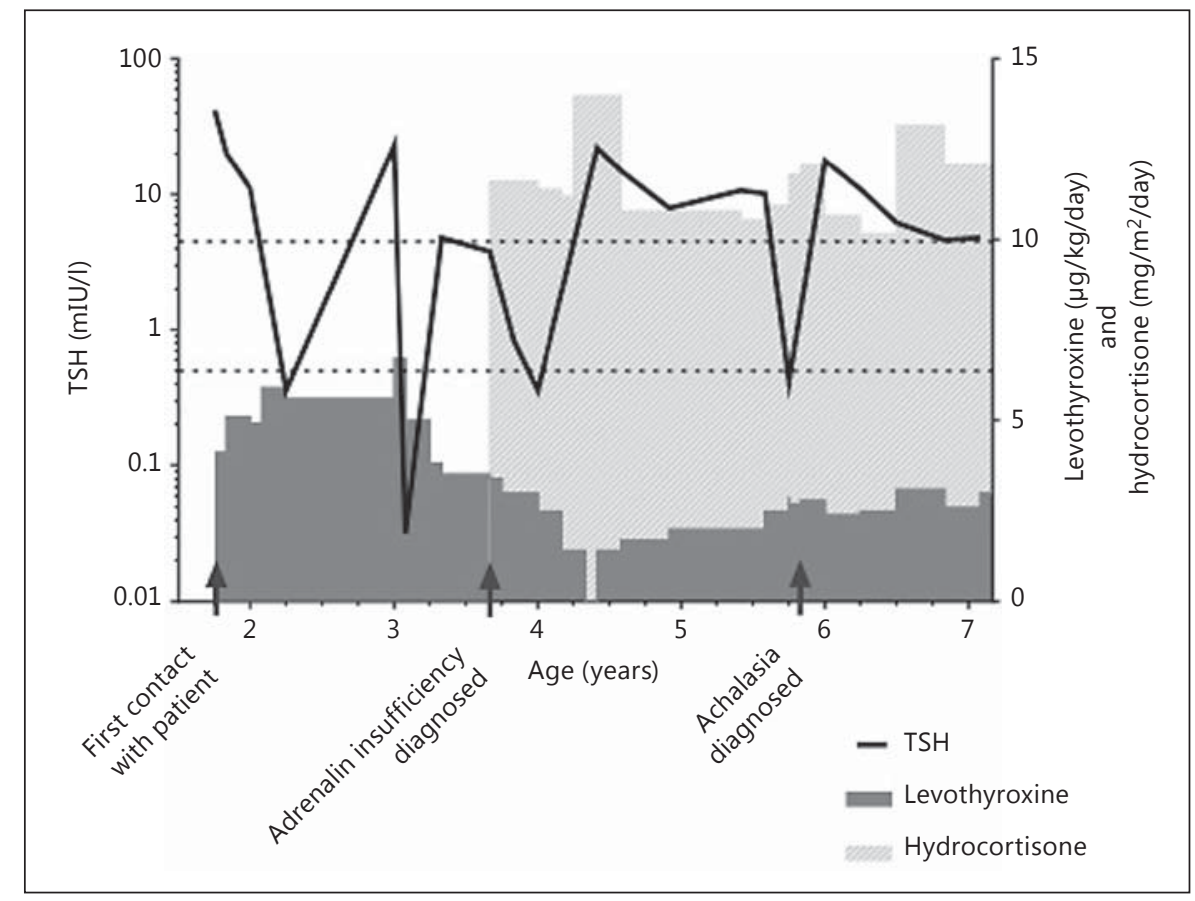

Pitfalls in Poorly Controlled Congenital Hypothyroidism
Horm Res Paediatr 2016;86:420-424 DOI: $10.1159 / 000446604$ 
neck showed a eutopic, normally sized thyroid gland. To confirm the diagnosis of permanent $\mathrm{CHT}$, at the age of 3 years, the levothyroxine was stopped. This resulted in a TSH concentration of 22 $\mathrm{mIU} / \mathrm{l}$ and an FT4 concentration of $12.9 \mathrm{pmol} / \mathrm{l}$ within 4 weeks, for which the levothyroxine substitution was resumed. At that time, there was no clinical suspicion of adrenal insufficiency or malabsorption. Because of CHT with eutopic thyroid gland without goiter in a consanguineous family and persistently elevated TSH levels in combination with a (low-)normal FT4, TSH receptor gene analysis was performed. Compound heterozygosity for mutations in the TSH receptor gene on chromosome 14q31 was found. One, c.1657G >A (p.Ala553Thr), has previously been described as a known pathogenic mutation. The other mutation, c.734G $>$ T (p. Gly245Val), was not described in the literature before. Only the c. $1657 \mathrm{G}>\mathrm{A}$ mutation was identified in the mother (the father could not be tested). Her TSH level was $6.3 \mathrm{mIU} / \mathrm{l}$ and her FT4 level was $15 \mathrm{pmol} / \mathrm{l}$.

Around the age of 3 years, both his height and weight for age were at the -2 SD line according to Dutch norms. His height was within the target height range. There was moderate global developmental delay, proximal and distal muscle weakness and, on electromyography, axonal polyneuropathy. The pediatric neurologist was unable to make a diagnosis and attributed the symptoms to the suboptimal thyroidal state.

At the age of 4 years, the boy presented with acute unconsciousness at the emergency department of a regional hospital. In the days prior to presentation, there was diarrhea and lack of appetite. His mother reported that there had been multiple episodes of subconsciousness in the past years, with spontaneous recovery. On presentation, our patient had lost consciousness. He was responsive only to pain, compatible with a Glasgow Coma Scale score of 6 points. He had a respiratory rate of $22 / \mathrm{min}$, a heart rate of $120 /$ min, a peripheral oxygen saturation of $97 \%$ and a core temperature of $35.7^{\circ} \mathrm{C}$. His lips were dark and there was mild hyperpigmentation especially in the skin folds. The bedside glucose concentration was below the detection limit of $0.1 \mathrm{mmol} / \mathrm{l}$. After an intravenous glucose bolus, his glucose reached low-normal values and, simultaneously, his clinical condition improved.

Laboratory evaluation showed a serum sodium concentration of $136 \mathrm{mmol} / \mathrm{l}$ and potassium of $3.6 \mathrm{mmol} / \mathrm{l}$. Ketone bodies were present in urine. His thyroid function was normal, with a TSH of $3.8 \mathrm{mIU} / \mathrm{l}$ and an FT4 of $17 \mathrm{pmol} / \mathrm{l}$. An undetectable cortisol concentration was found $(<10 \mathrm{nmol} / \mathrm{l})$, which was accompanied by an elevated adrenocorticotropin (ACTH) concentration $(>1,250$ $\mathrm{ng} / \mathrm{l}$ ), while renin and aldosterone concentrations were normal. An ACTH stimulation test confirmed the suspicion of primary hypocortisolism, with cortisol concentrations that remained undetectable $(<0.01 \mu \mathrm{mol} / \mathrm{l}) 30$ and $60 \mathrm{~min}$ after administration of $250 \mu \mathrm{g} \mathrm{ACTH} \mathrm{A}_{1-24}$. These findings were indicative for an Addisonian crisis of adrenal origin, and treatment with hydrocortisone was started instantly $\left(50 \mathrm{mg} / \mathrm{m}^{2} /\right.$ day initially, thereafter $11 \mathrm{mg} / \mathrm{m}^{2}$ / day), with rapid clinical improvement.

After the start of the hydrocortisone treatment, the boy showed an improvement in psychomotor development as well as a normalization of TSH, for which his levothyroxine could be lowered by fifty percent. Furthermore, the hyperpigmentation disappeared. Additional investigations for the cause of the hypocortisolism were performed. Serum analysis for inborn errors of metabolism (e.g. long-chain fatty acids for diagnosing adrenoleukodystrophy) showed no abnormalities, and antibodies directed against the ad- renal gland were not present. Extensive DNA analysis showed no abnormalities in the $D A X-1$ (dosage-sensitive sex reversal, adrenal hypoplasia critical region, on chromosome X, gene 1), SF-1 (splicing factor 1), $M C 2 R$ (melanocortin-2 receptor), MRAP (melanocortin-2 receptor accessory protein) and NNT (nicotinamide nucleotide transhydrogenase) genes.

After a brief period of improvement, the motor function gradually declined again. Magnetic resonance imaging of the brain showed mild cerebral and cerebellar atrophy and enhanced signal intensity bilateral in the occipital region, probably due to former hypoglycemic episodes. At physical examination, progressive muscle weakness was noticed, with a positive Gower sign and increased signal intensity especially in the lower limbs on muscle ultrasound. A muscle biopsy was scheduled, but meanwhile, the boy developed gastrointestinal symptoms consisting of frequent vomiting of small amounts of old meals, continuous appetite but inability to eat more than a few spoons at once, $5 \mathrm{~kg}$ of weight loss in 4 months and tooth decay, for which all of his primary teeth had to be extracted. In this period, his TSH increased again, even after the levothyroxine dosage was raised. At the age of 6 years, he was admitted to the hospital in a malnourished state. An X-ray with oral contrast showed difficult passage of contrast from esophagus to stomach with stasis in the distal esophagus and a dilated aspect of the whole esophagus. Esophageal manometry confirmed the diagnosis of achalasia, after which balloon dilatation was performed.

The combination of adrenal insufficiency and achalasia directed us towards the diagnosis of the triple-A syndrome. In retrospect, our patient also exhibited alacrima and the neurological symptoms could fit into the fourth A of amyotrophy. Wholeexome sequencing confirmed the diagnosis of Allgrove syndrome by the discovery of a homozygous mutation in the AAAS gene [c.13311+1G>A p.(?) (NM_015665.5), which is a known founder mutation (OMIM 605378.0005)]. The same mutation was found in a heterozygous form in the mother (again, the father could not be tested). With the diagnosis of Allgrove syndrome, the CHT could subsequently be considered as a separate disease entity.

\section{Discussion}

CHT is a major cause of preventable developmental delay if left untreated. In most cases of primary $\mathrm{CHT}$, TSH is easily normalized after substitution with levothyroxine. In case of persistently elevated TSH concentrations, maladministration must be evaluated, but comorbid conditions such as malabsorption and adrenal insufficiency may also be possible [2]. When a patient has a persistently elevated TSH concentration, in spite of adequate levothyroxine dosage and intake, other causes must be explored.

Resistance to TSH due to TSH receptor (TSHR) mutations is increasingly recognized as a cause of CHT [3]. In 2010, Cangul et al. [4] reported that amongst 139 children with autosomal recessive nongoitrous CHT born to consanguineous families, 5\% had a homozygous, biallelic inactivation of the TSHR. TSHR mutations show a strong 
genotype-phenotype correlation. However, the clinical presentation of children with TSHR mutations ranges from only mild TSH elevation without any clinical features to severe hypothyroidism with developmental delay, failure to thrive and short stature [5]. In this case, two different TSHR mutations were found, probably both pathogenic. In patients with TSHR mutations, TSH levels usually normalize after starting levothyroxine in a substitution dosage, but there is ongoing debate about whether treatment is warranted in all of them.

The triad of adrenal insufficiency, achalasia and absent lacrimation - the triple-A or Allgrove syndrome - was first described by Allgrove et al. in 1978 [6]. More than a decade later, it was reported that some patients with this syndrome have a fourth $\mathrm{A}$, which refers to amyotrophy $[7,8]$. Nowadays, Allgrove syndrome is increasingly considered as a multisystem disease, with gradual onset of symptoms that often leads to diagnostic delay. In 2000, it was discovered that mutations in the AAAS gene on chromosome 12q13 (encoding the Aladin protein) underlie Allgrove syndrome [9]. Allgrove syndrome typically presents in childhood with dysphagia or hypoglycemia (due to adrenal crisis) as first symptom [10], although it has also been described to present as late as in adulthood with neurological symptoms as presenting sign [11]. Alacrima is retrospectively often the first and most specific finding, but is easily neglected [12].

In our case, the multiple nonspecific neurological symptoms were probably the first sign of Allgrove syndrome, but it was only after the adrenal insufficiency and the achalasia appeared that specific gene analysis was performed. Delayed diagnosis is common in Allgrove syndrome [12]. Thyroid dysfunction is not part of Allgrove syndrome, and although TSH concentrations may have been influenced by the coexistence of adrenal insufficiency, the CHT in this boy must be considered as a separate entity.

Our case had multiple pitfalls. First, the presence of the CHT due to mild TSH resistance and the assumption that the persistent increase in TSH was most likely caused by malcompliance to levothyroxine therapy. Eventually, the persistent TSH elevation in our patient could be attributed to hypocortisolism and malabsorption of levothyroxine. Although cortisol inhibits the release of TSH by the pituitary gland [2], adrenal insufficiency was not the primary suspect in our case, as signs of hypocortisolism were not manifest at birth. This is in line with the pathogenesis of adrenal insufficiency in Allgrove syndrome, which is likely to be caused by a decreased oxidative stress response that becomes manifest later in childhood [13].
The fact that even after the start of hydrocortisone the boy still had frequent episodes of TSH elevation argued for another cause. Finally, achalasia, with recurrent regurgitation and progressive stasis of ingested food in the esophagus, was found.

The second pitfall was the relatively late discovery of Allgrove syndrome as the cause of the hypocortisolism. Possibly, the chosen stepwise diagnostic approach for the adrenal insufficiency contributed to this delay. First, more common causes like congenital adrenal hyperplasia, autoimmune adrenalitis and adrenoleukodystrophy were excluded. In retrospect, earlier analysis of recessive genes could have been arranged, as parents were known to be consanguineous.

In the third place, the other A's, of alacrima and achalasia, of Allgrove syndrome should have received more attention. In retrospect, the tooth decay was a presenting symptom of achalasia, already before the weight loss and food passage problems. None of the different medical doctors specifically asked about dryness of the eyes or absence of tears with crying. The mother did not know that this was an important key in the diagnosis, so it was never spontaneously reported. Neurological examination was extensively repeated, but the many distinct features (of neuropathy, muscle weakness and neurocognitive impairment) were not recognized as part of Allgrove syndrome.

In conclusion, this case illustrates the many pitfalls that may occur in diagnosing a rare syndrome. Furthermore, when treatment of a patient with congenital hypothyroidism or mild TSH resistance is unexplainably difficult and accompanying symptoms are present which cannot be fully attributed to the thyroid disease, a new and broader differential diagnosis should be actively looked for. This may diminish doctor's and patient's delay.

\footnotetext{
References

1 Léger J: Congenital hypothyroidism: a clinical update of long-term outcome in young adults. Eur J Endocrinol 2015;172:R67-R77.

2 Nicoloff JT, Fisher DA, Appleman MD Jr: The role of glucocorticoids in the regulation of thyroid function in man. J Clin Invest 1970; 49:1922-1929.

3 Park SM, Chatterjee VK: Genetics of congenital hypothyroidism. J Med Genet 2005;42: 379-389.
} 
4 Cangul H, Morgan NV, Forman JR, Saglam $\mathrm{H}$, Aycan Z, Yakut T, Gulten T, Tarim O, Bober E, Cesur Y, Kirby GA, Pasha S, Karkucak M, Eren E, Cetinkaya S, Bas V, Demir K, Yuca SA, Meyer E, Kendall M, Hogler W, Barrett TG, Maher ER: Novel TSHR mutations in consanguineous families with congenital nongoitrous hypothyroidism. Clin Endocrinol 2010;73:671-677.

5 Kopp P: The TSH receptor and its role in thyroid disease. Cell Mol Life Sci 2001;58:13011322.

6 Allgrove J, Clayden GS, Grant DB, Macaulay JC: Familial glucocorticoid deficiency with achalasia of the cardia and deficient tear production. Lancet 1978;1:1284-1286.
7 Grant DB, Dunger DB, Smith I, Hyland K: Familial glucocorticoid deficiency with achalasia of the cardia associated with mixed neuropathy, long-tract degeneration and mild dementia. Eur J Pediatr 1992;151:85-89.

8 Gazarian M, Cowell CT, Bonney M, Grigor WG: The ' $4 \mathrm{~A}$ ' syndrome: adrenocortical insufficiency associated with achalasia, alacri$\mathrm{ma}$, autonomic and other neurological abnormalities. Eur J Pediatr 1995;154:18-23.

9 Tullio-Pelet A, Salomon R, Hadj-Rabia S, Mugnier C, de Laet MH, Chaouachi B, Bakiri F, Brottier P, Cattolico L, Penet C, Bégeot M, Naville D, Nicolino M, Chaussain JL, Weissenbach J, Munnich A, Lyonnet S: Mutant WD-repeat protein in triple-A syndrome. Nat Genet 2000;26:332-335.
10 Kimber J, McLean BN, Prevett M, Hammans SR: Allgrove or 4 'A' syndrome: an autosomal recessive syndrome causing multisystem neurological disease. J Neurol Neurosurg Psychiatry 2003;74:654-657.

11 Vallet AE, Verschueren A, Petiot P, Vandenberghe N, Nicolino M, Roman S, Pouget J, Vial C: Neurological features in adult Triple-A (Allgrove) syndrome. J Neurol 2012;259:39-46.

12 Kesar PA, Khadilkar VV, Tibrewala VN: Allgrove syndrome. Indian J Pediatr 2007;74: 959-961.

13 Jühlen R, Idkowiak J, Taylor AE, Kind B, Arlt W, Huebner A, Koehler K: Role of ALADIN in human adrenocortical cells for oxidative stress response and steroidogenesis. PLoS One 2015;10:e124582. 